\title{
Copper Oxide Micro-Tufts on Natural Fractals for Efficient Water Harvesting
}

\author{
Vipul Sharma ${ }^{1 *}$, Harri Ali-Löytty ${ }^{2}$, Anastasia Koivikko ${ }^{1}$, Kyriacos Yiannacou ${ }^{1}$, Kimmo Lahtonen $^{3}$ \\ and Veikko Sariola ${ }^{1 *}$ \\ ${ }^{1}$ Faculty of Medicine and Health Technology, Tampere University, Finland \\ Korkeakoulunkatu 3, 33720 Tampere, Finland \\ ${ }^{2}$ Surface Science Group, Photonics Laboratory, Tampere University, P.O. Box 692, FI-33014 \\ Tampere University, Finland
}

${ }^{3}$ Faculty of Engineering and Natural Sciences, Tampere University,

P.O. Box 692, 33014 Tampere, Finland

*Email: vipul.sharma@tuni.fi,veikko.sariola@tuni.fi

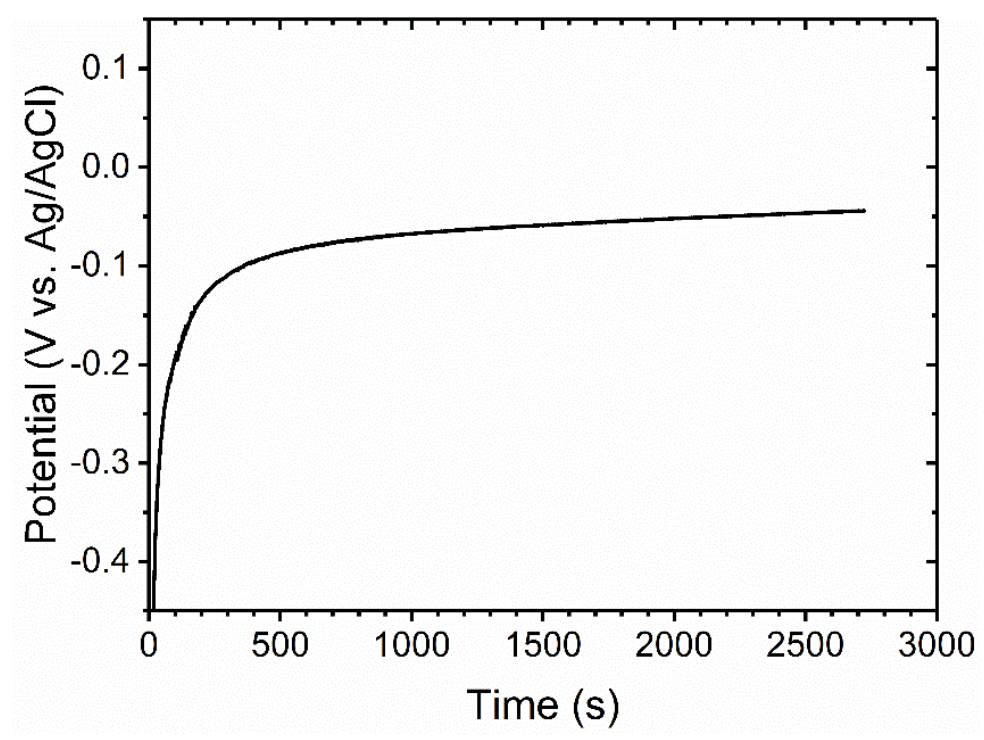

Figure S1. Potential as a function of time during galvanostatic electrodeposition of $\mathrm{Cu}$ on Au coated leaf skeleton at $-10 \mathrm{~mA} / \mathrm{cm}^{2}$. 

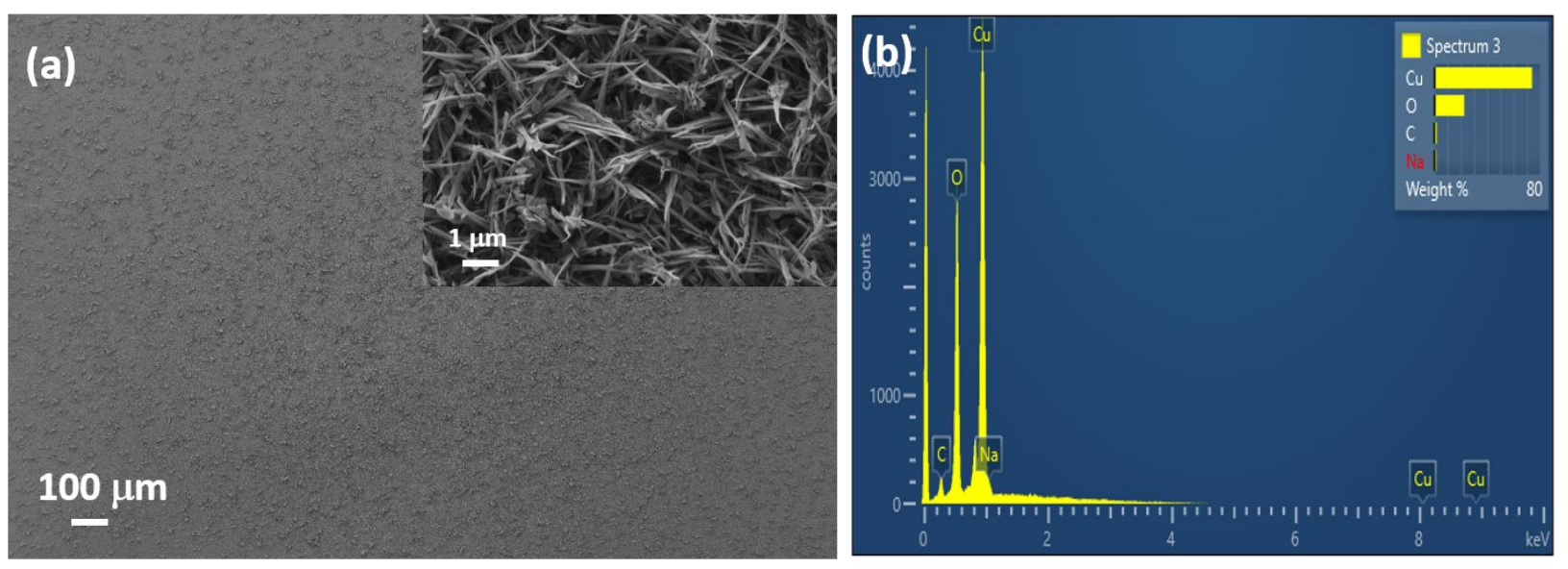

Figure S2. (a) FESEM image of the CuO grown on the glass substrate (planer surface). (b) Shows the corresponding EDS spectra. 

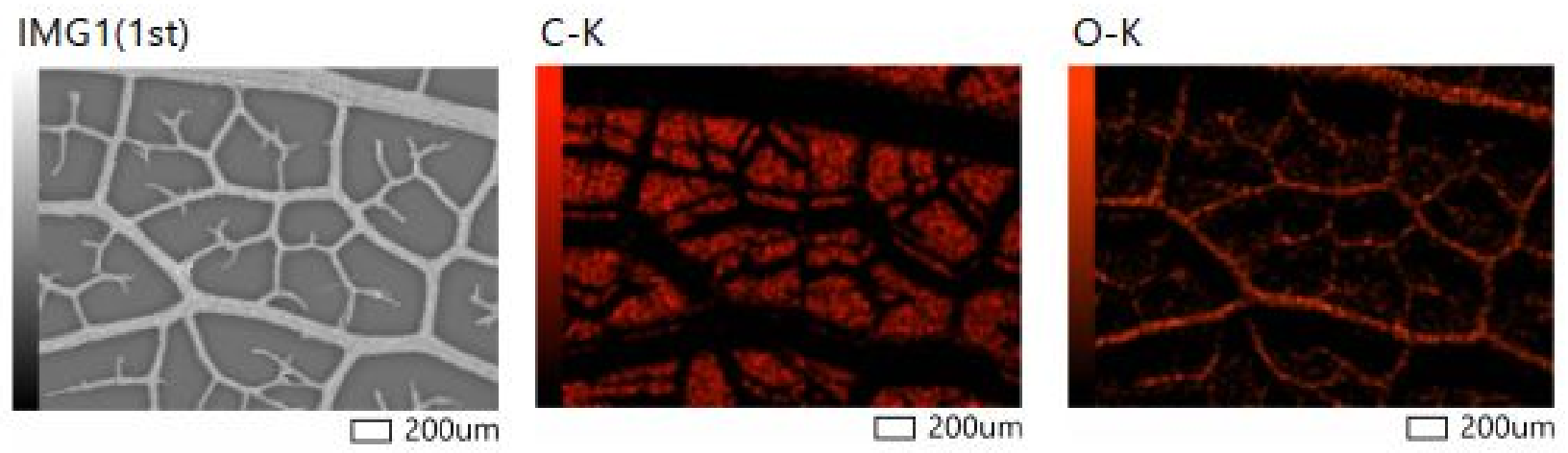

$\mathrm{Na}-\mathrm{K}$

$\mathrm{Ca}-\mathrm{K}$

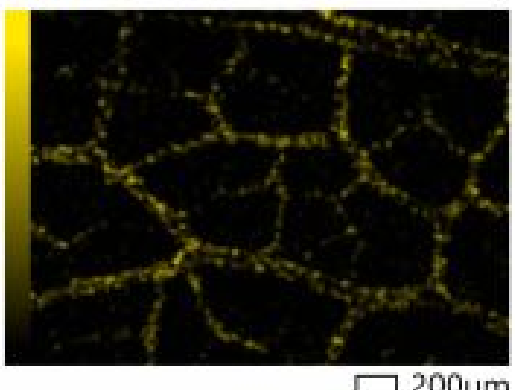

Au-M

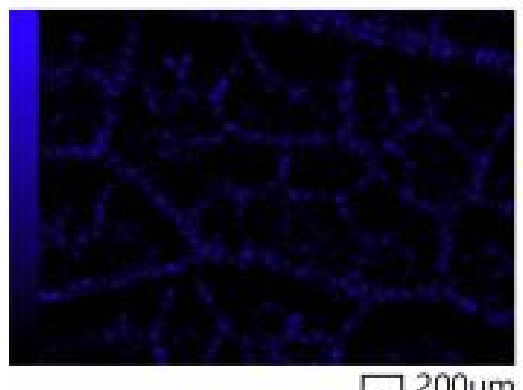

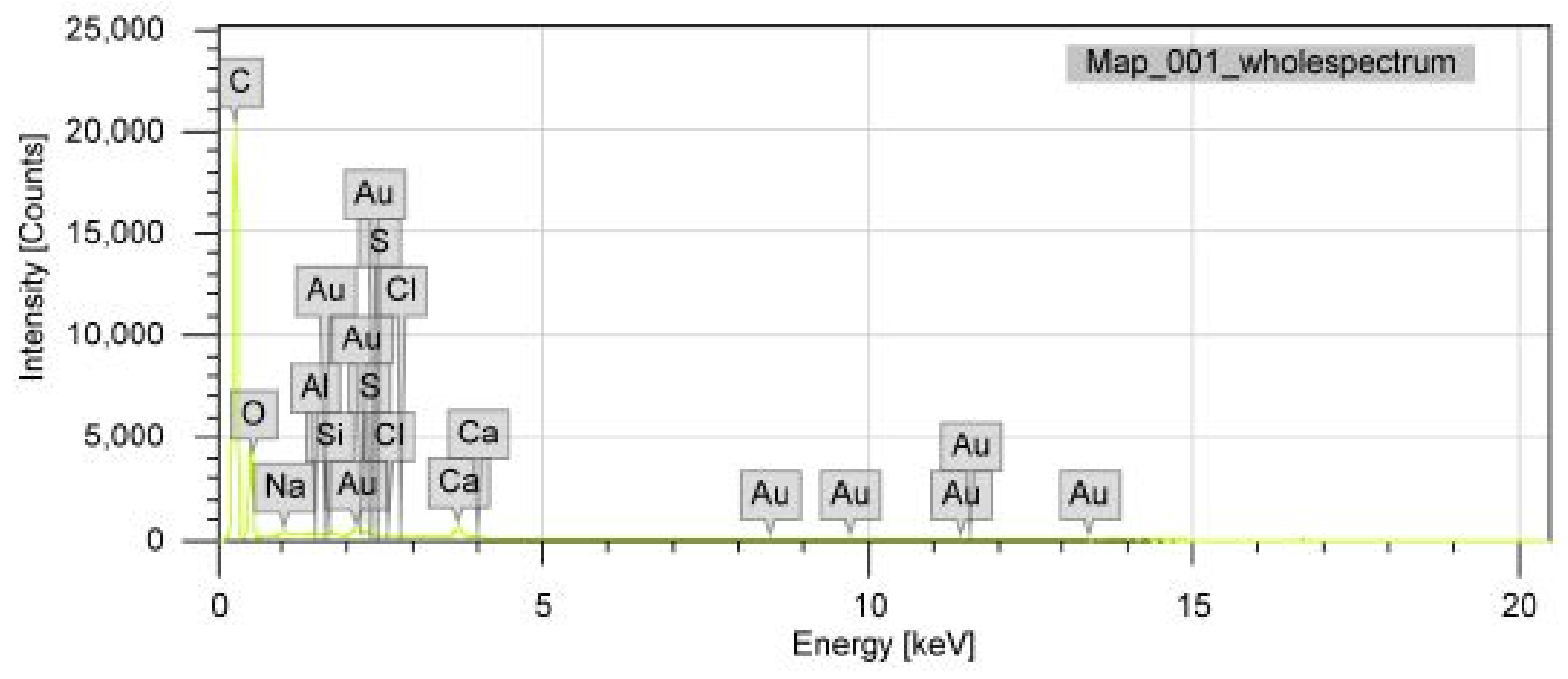

Figure S3. EDS mapping and corresponding spectra of the leaf skeleton after Au deposition. 


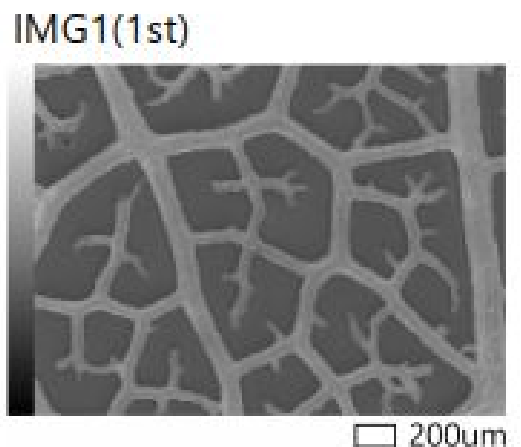

IMG1

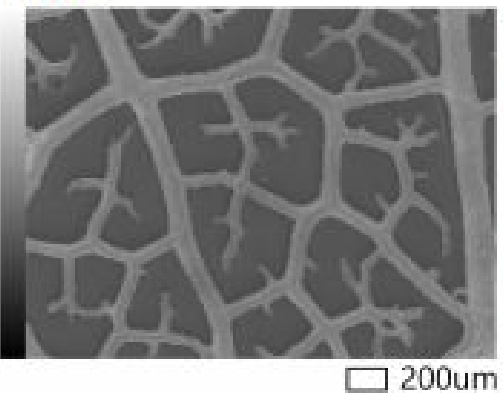

$\mathrm{O}-\mathrm{K}$

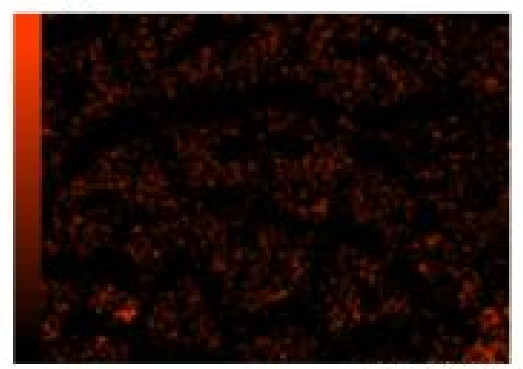

$\square 200 \mathrm{um}$
S-K

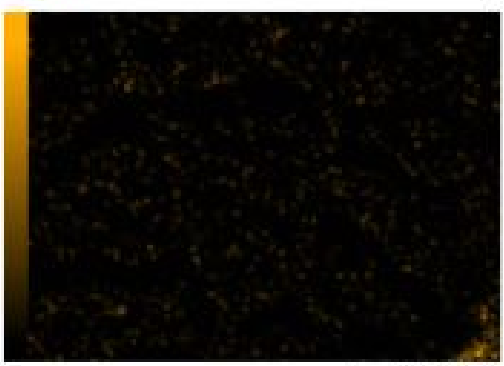

200um
C-K

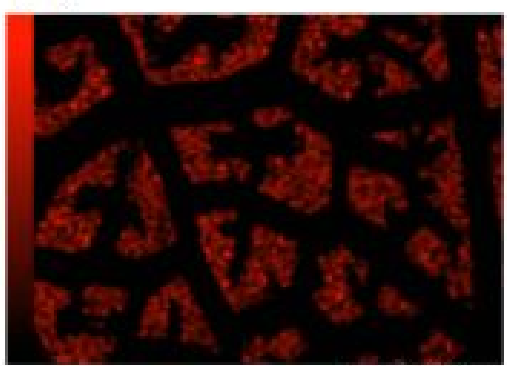

200um

$\mathrm{Cu}-\mathrm{K}$

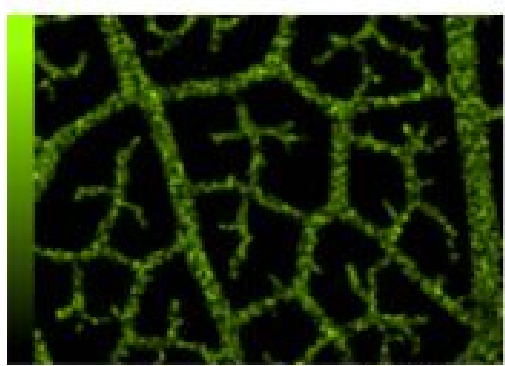

200um

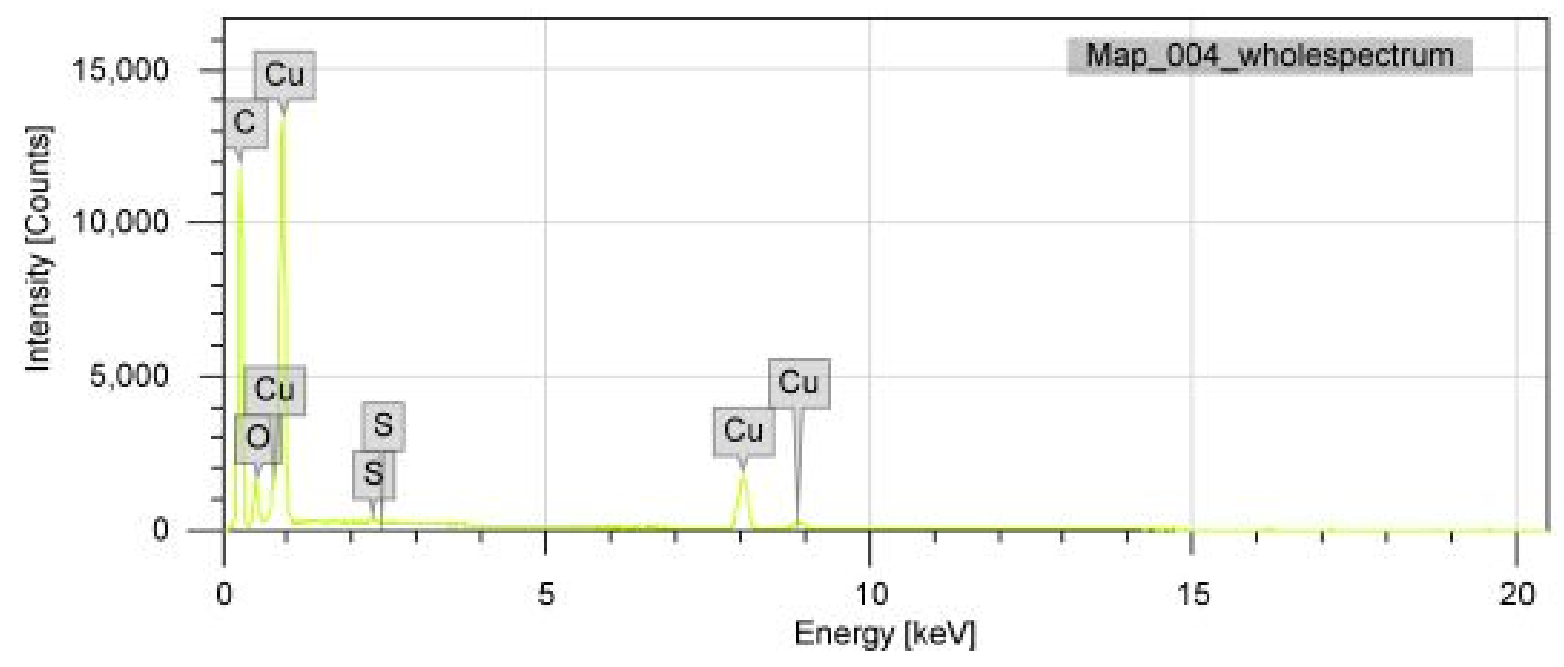

Figure S4. EDS mapping and corresponding spectra of the leaf skeleton after Cu deposition. 


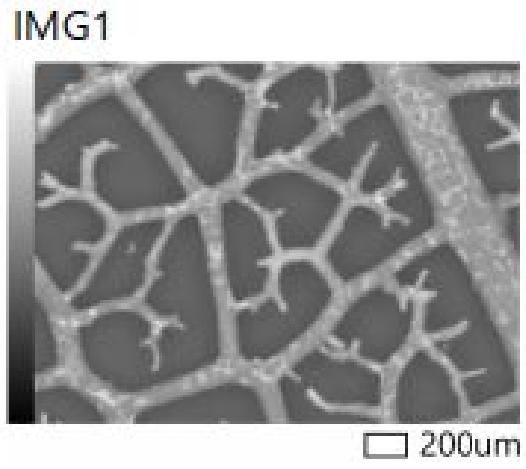

$\mathrm{Na}-\mathrm{K}$

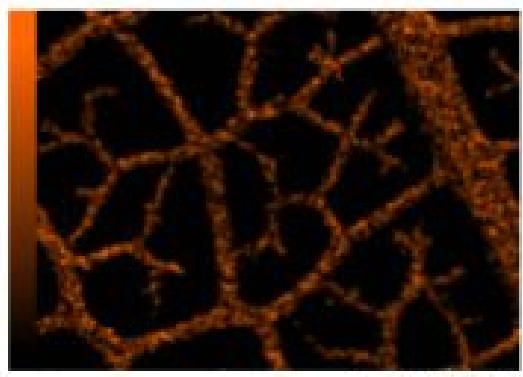

$\square$ 200um
C-K

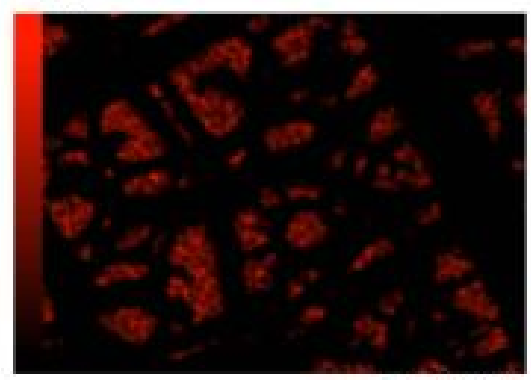

200um

S-K

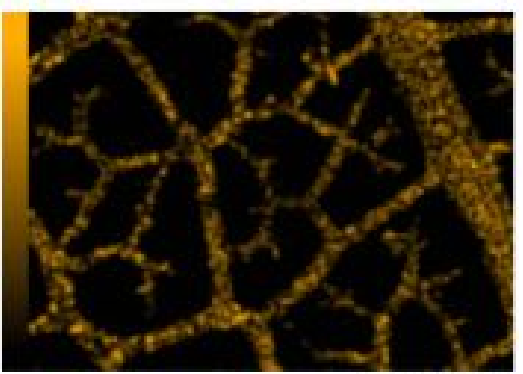

200um
O-K

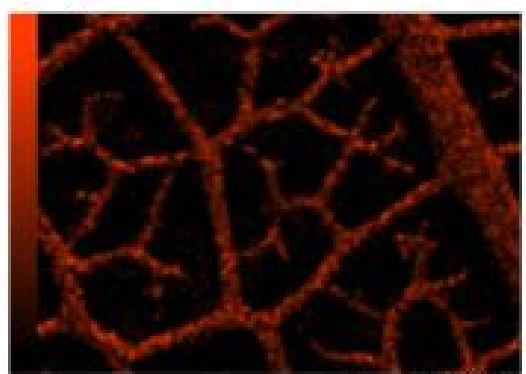

$\square$ 200um

Cu-K

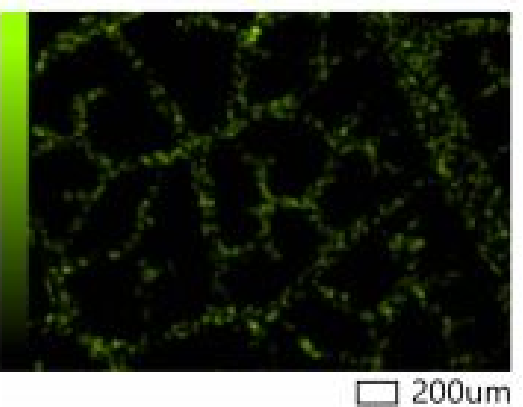

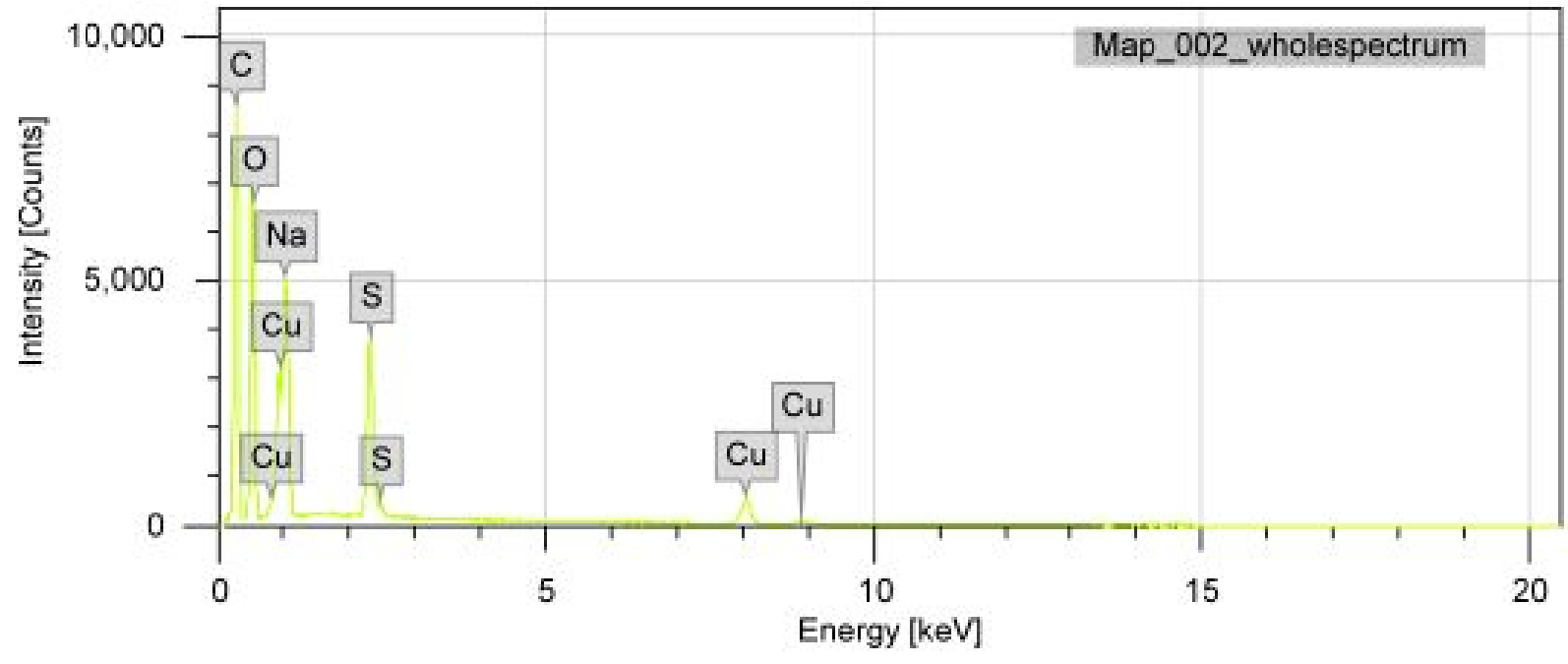

Figure S5. EDS mapping and corresponding spectra of the leaf skeleton after CuO microtuft fabrication on the surface via oxidation. 


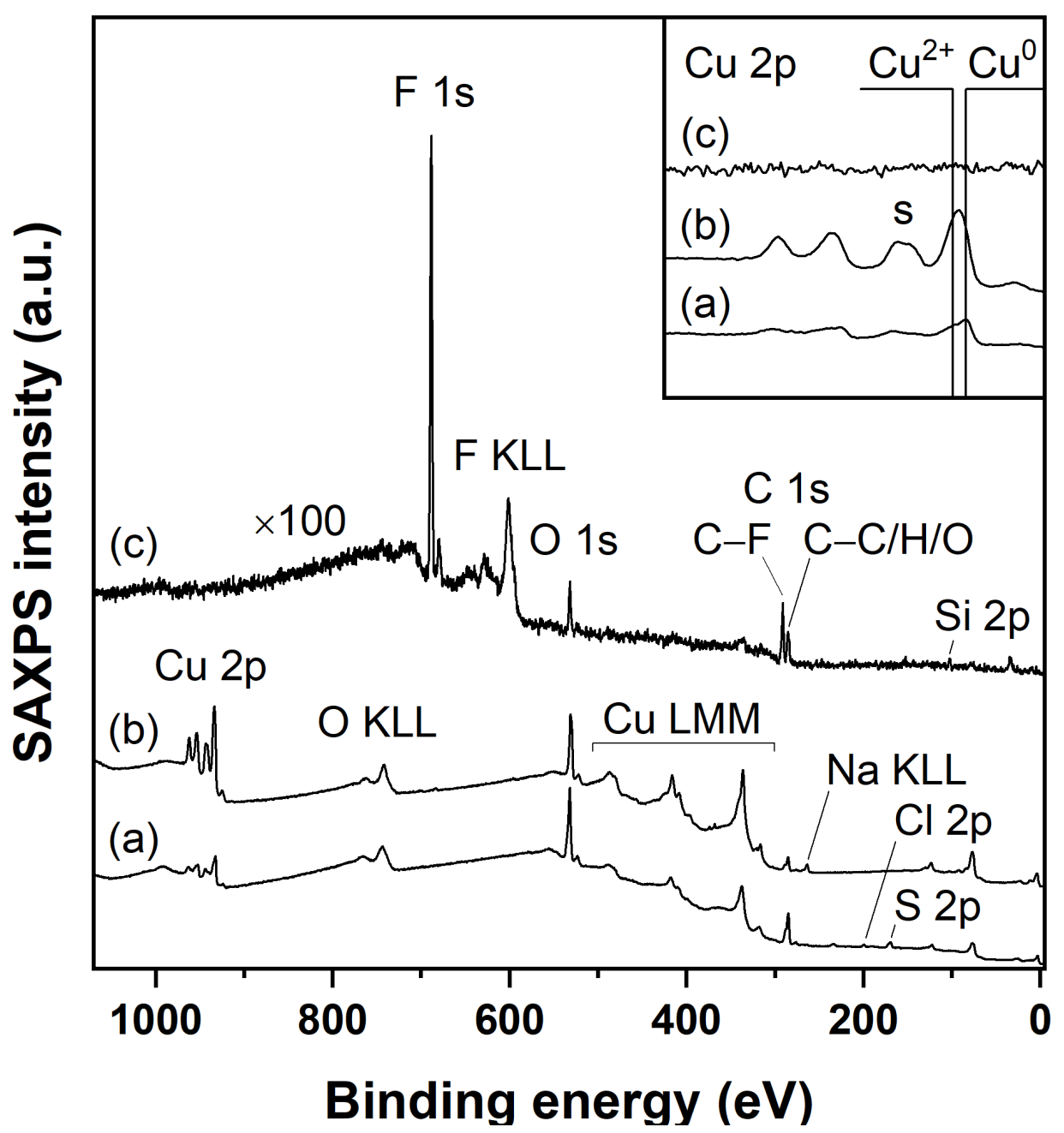

Figure S6. SAXPS survey spectra of a. $\mathrm{Cu}, \mathbf{b}$. $\mathrm{CuO}$, and c. CuO silane (intensity $\times 100$ ). The inset shows zoom-in of the $\mathrm{Cu} 2 \mathrm{p}$ region where " $\mathrm{s}$ " $=\mathrm{Cu}^{2+}$ shakeup. Note the low intensity and high noise level in c. Low X-ray flux was used to scan the spectrum because of X-ray induced outgassing (C-F bond breaking) of the sample. 

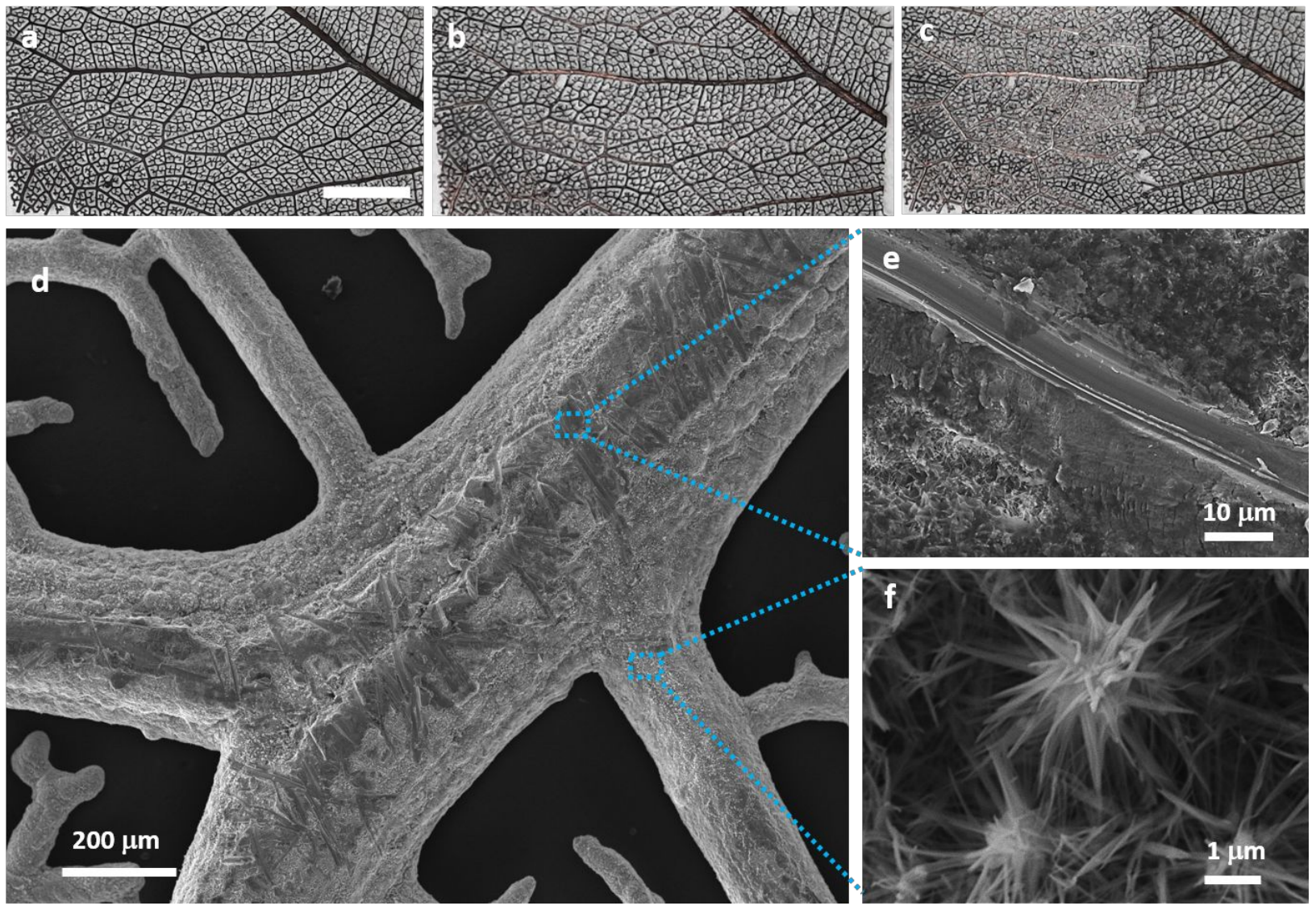

Figure S7. Camera images of the surfaces, a. before scoring with sandpaper, $\mathbf{b}$. after mild abrasion (25 cycles) and c. heavy abrasion. The scalebar in camera images is $5 \mathrm{~mm}$. d. shows the SEM image of the abraded sample ( 25 cycles). e. and $\mathbf{f}$. show the areas with the abrasion signs and the shielded areas with the microtufts, respectively. 


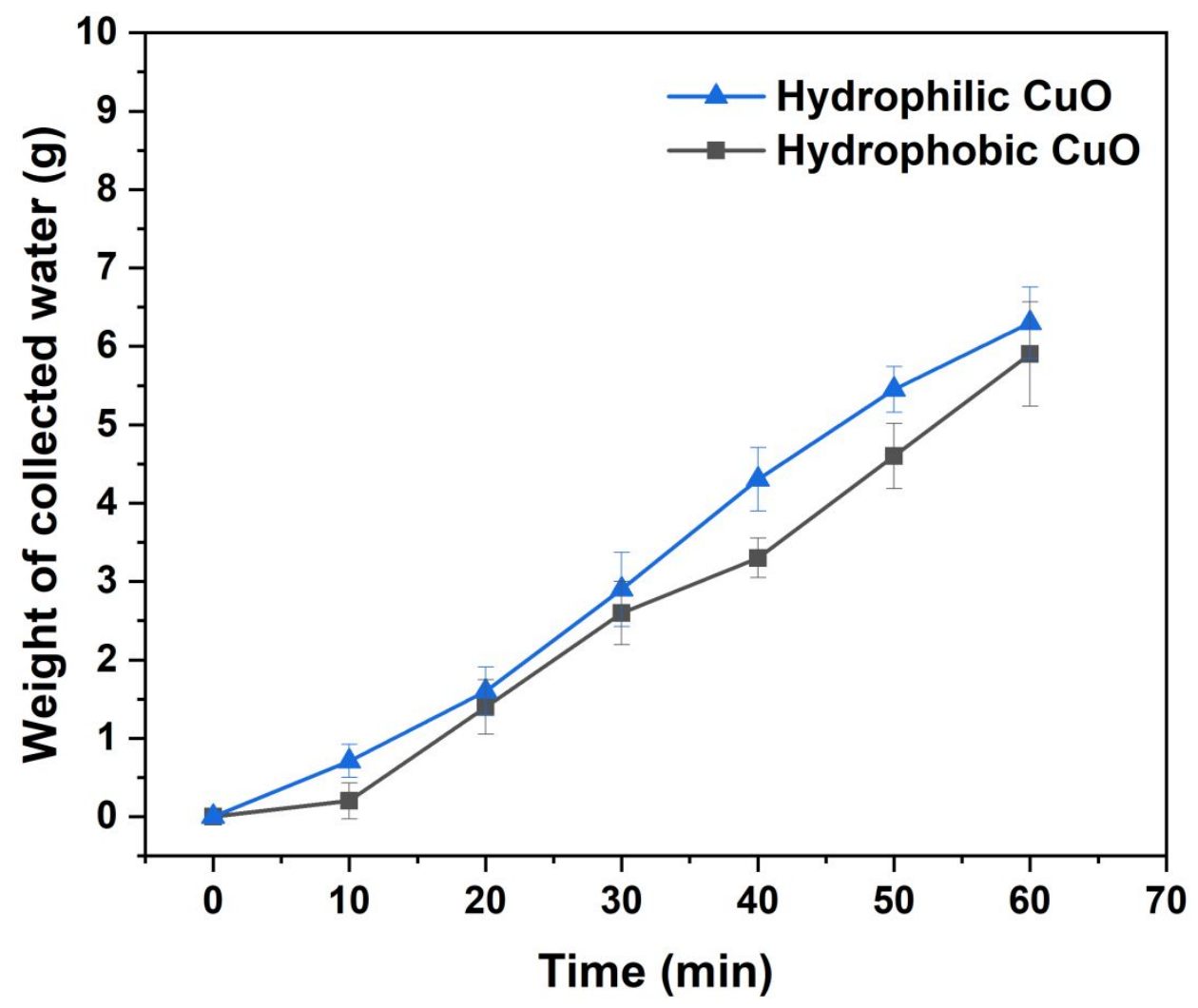

Figure S8. Fog harvesting dynamics as the volume of water collected $(\mathrm{g})$ in time, $\mathrm{t}(\mathrm{min})$, over abraded skeleton surfaces (scored with sandpaper, 25 cycles) having dimensions $40 \times 40 \mathrm{~mm}$. Error bars show the standard deviations calculated from three independent measurements. 

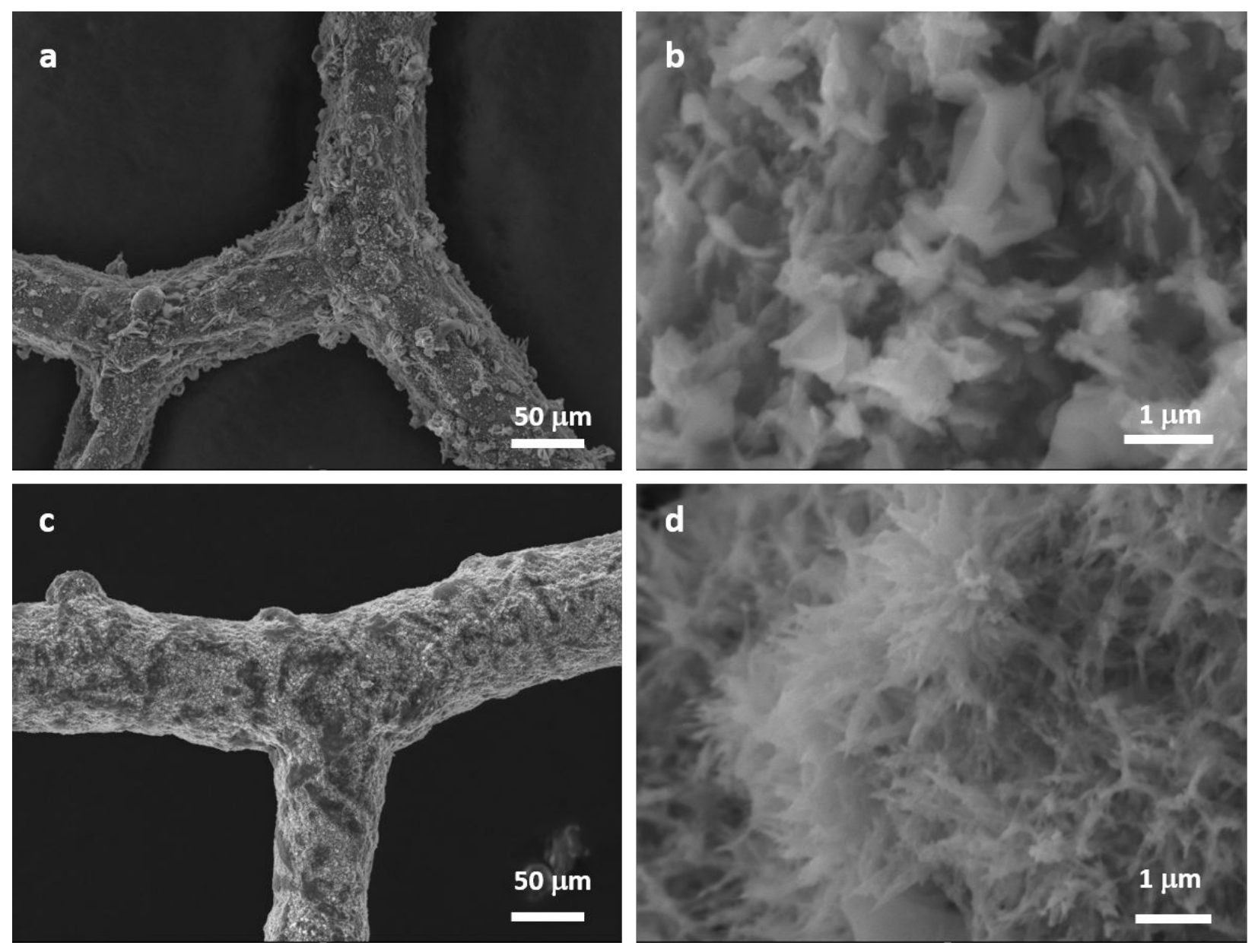

Figure S9. SEM Images of the 100 days old leaf skeleton bearing (a-b), CuO microtufts without silane coating, and (c-d) CuO microtufts with silane coating. 


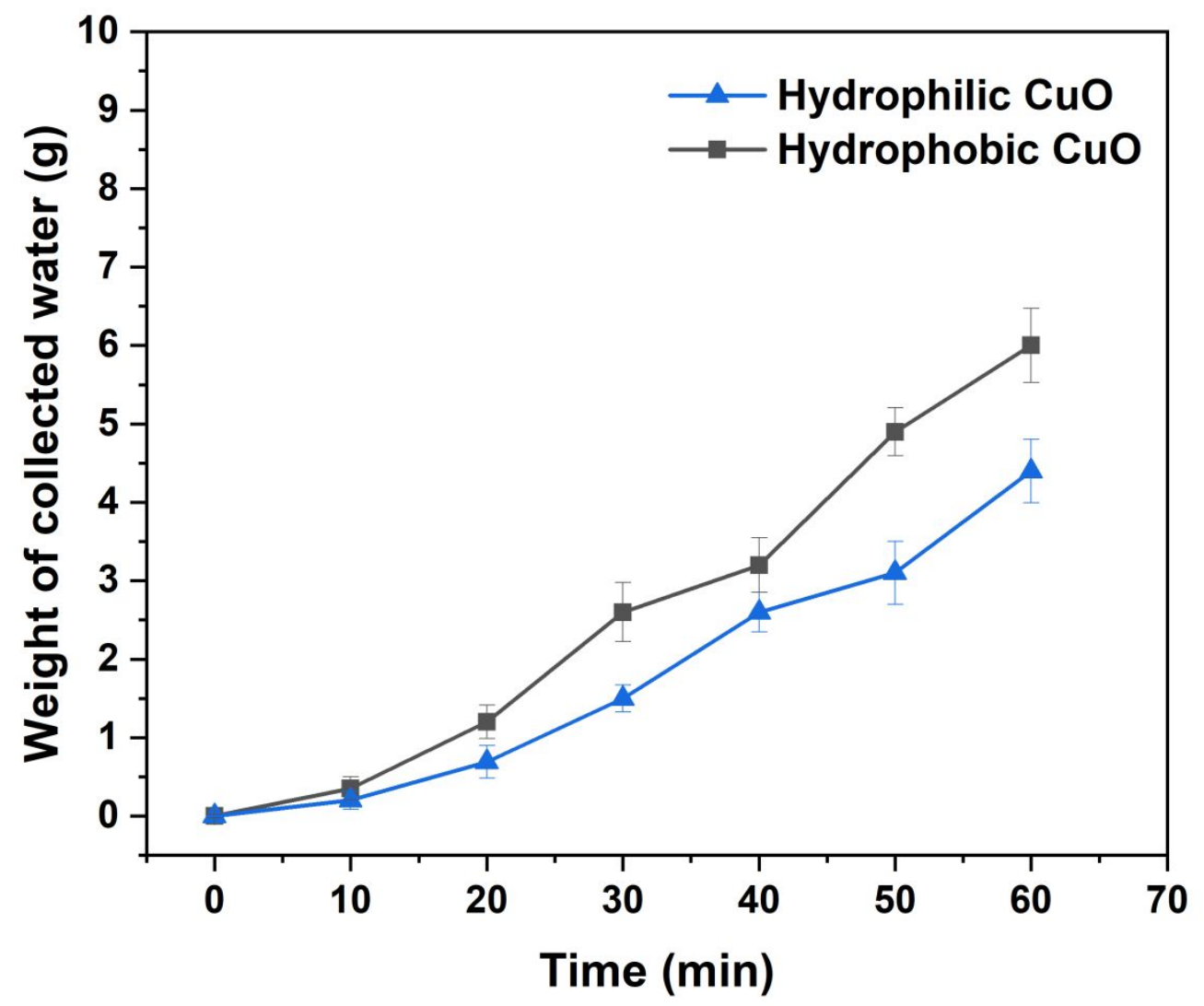

Figure S10. Fog harvesting dynamics as volume of water collected $(\mathrm{g})$ in time, $t(\mathrm{~min})$, over 100 days old skeleton surfaces having dimensions $40 \times 40 \mathrm{~mm}$. Error bars show the standard deviations calculated from three independent measurements.

Table S1. Analysis of variance.

\begin{tabular}{|c|c|c|c|c|c|}
\hline Source & Sum Sq. & d.f. & Mean Sq. & F & Prob>F \\
\hline Direction & 0.5652 & 3 & 0.1884 & 29.9 & 0.0098 \\
\hline Hydrophobicity & 2.1219 & 1 & 2.1219 & 336.71 & 0.0004 \\
\hline CuO/ Cu & 31.8472 & 1 & 31.8472 & 5053.63 & 0 \\
\hline Direction (Hydrophobic) & 0.982 & 3 & 0.3273 & 51.94 & 0.0044 \\
\hline Direction (CuO/Cu) & 0.565 & 3 & 0.0188 & 2.99 & 0.1962 \\
\hline Hydrophobicity (CuO/Cu) & 0.747 & 1 & 0.747 & 11.86 & 0.0411 \\
\hline
\end{tabular}




\begin{tabular}{|l|c|c|c|c|c|}
\hline Error & 0.0189 & 3 & 0.0063 & & \\
\hline Total & 35.6664 & 15 & & & \\
\hline
\end{tabular}

Table S2. Comparison of fog collection performance of different Cu-based surfaces reported in the literature.

\begin{tabular}{|c|c|c|c|c|}
\hline $\begin{array}{l}\text { S. } \\
\text { No. }\end{array}$ & Fabrication Method & $\begin{array}{c}\text { Fog flow } \\
\text { rate }\left(\mathrm{cm} \mathrm{s}^{-1}\right)\end{array}$ & $\begin{array}{l}\text { Water collection rate } \\
\qquad\left(\mathrm{g} \mathrm{cm}^{-2} \mathrm{~h}^{-1}\right)\end{array}$ & Ref. \\
\hline 1 & $\begin{array}{l}\text { Alternative thiol modification of } \\
\mathrm{TiO}_{2} \text { and } \mathrm{Cu}\end{array}$ & 10 & $\sim 1.309$ & 1 \\
\hline 2 & $\begin{array}{l}\text { Photocatalysis of } \mathrm{CuO} \text { on } \mathrm{a} \mathrm{TiO}_{2} \\
\text { coated copper mesh }\end{array}$ & 50 & $\sim 0.571$ & 2 \\
\hline 3 & $\begin{array}{l}\text { Chemical oxidation of scorched } \\
\text { Cu plate }\end{array}$ & 5-15 & $\sim 0.5$ & 3 \\
\hline 4 & $\begin{array}{l}\text { Chemically modified 1D Cu } \\
\text { wires }(\sim 50 \mu \mathrm{m})\end{array}$ & 60 & $\sim 11.6$ & 4 \\
\hline 5 & $\begin{array}{l}\text { Electrochemical deposition and } \\
\text { ammonia corrosion of } \mathrm{Cu}\end{array}$ & $\sim 25$ & $\sim 0.707$ & 5 \\
\hline 6 & $\begin{array}{l}\text { Ammonia corrosion of } \\
\mathrm{Cu} / \text { liquidus modification }\end{array}$ & $\sim 50$ & $\sim 1.3$ & 6 \\
\hline 7 & Electrochemical corrosion & 240 & $\sim 0.618$ & 7 \\
\hline 8 & $\begin{array}{l}\text { Ammonia corrosion / particle } \\
\text { deposition }\end{array}$ & $50-70$ & 0.430 & 8 \\
\hline 9 & Alkaline oxidation/modification & N/A & 1.317 & 9 \\
\hline 10 & Chemically modified $\mathrm{Cu}$ & 25 & $\sim 0.211$ & 10 \\
\hline
\end{tabular}




\begin{tabular}{|c|l|c|c|c|}
\hline 11 & $\begin{array}{l}\text { Electrodeposited CuO microtufts } \\
\text { on leaf skeletons }\end{array}$ & $5-15$ & 0.45 & $\begin{array}{c}\text { This } \\
\text { work }\end{array}$ \\
\hline
\end{tabular}

\section{References:}

(1) Zhu, H.; Guo, Z. Hybrid Engineered Materials with High Water-Collecting Efficiency Inspired by Namib Desert Beetles. Chem. Commun. 2016, 52 (41), 6809-6812.

(2) Gou, X.; Guo, Z. Hybrid Hydrophilic-Hydrophobic CuO@TiO 2-Coated Copper Mesh for Efficient Water Harvesting. Langmuir 2019, 36, 1, 64-73.

(3) Sharma, V.; Yiannacou, K.; Karjalainen, M.; Lahtonen, K.; Valden, M.; Sariola, V. Large-Scale Efficient Water Harvesting Using Bioinspired Micro-Patterned Copper Oxide Nanoneedle Surfaces and Guided Droplet Transport. Nanoscale Adv. 2019, 1, 4025-4040.

(4) Zhong, L.; Zhang, R.; Li, J.; Guo, Z.; Zeng, H. Efficient Fog Harvesting Based on 1D Copper Wire Inspired by the Plant Pitaya. Langmuir 2018, 34 (50), 15259-15267.

(5) Zhou, H.; Jing, X.; Guo, Z. Optimal Design of a Fog Collector: Unidirectional Water Transport on a System Integrated by Conical Copper Needles with Gradient Wettability and Hydrophilic Slippery Rough Surfaces. Langmuir 2020. 36, 6801-6810.

(6) Zhong, L.; Feng, J.; Guo, Z. An Alternating Nanoscale (Hydrophilic-Hydrophobic)/Hydrophilic Janus Cooperative Copper Mesh Fabricated by a Simple Liquidus Modification for Efficient Fog Harvesting. J. Mater. Chem. A 2019, 7 (14), 8405-8413.

(7) Xu, T.; Lin, Y.; Zhang, M.; Shi, W.; Zheng, Y. High-Efficiency Fog Collector: Water Unidirectional Transport on Heterogeneous Rough Conical Wires. ACS Nano 2016, 10 (12), 10681-10688.

(8) Zhong, L.; Zhu, H.; Wu, Y.; Guo, Z. Understanding How Surface Chemistry and Topography Enhance Fog Harvesting Based on the Superwetting Surface with Patterned Hemispherical Bulges. J. Colloid Interface Sci. 2018, 525, 234-242.

(9) Zhu, H.; Yang, F.; Li, J.; Guo, Z. High-Efficiency Water Collection on Biomimetic Material with Superwettable Patterns. Chem. Commun. 2016, 52 (84), 12415-12417. 
(10) Zhou, H.; Jing, X.; Guo, Z. Excellent Fog Droplets Collector via an Extremely Stable Hybrid Hydrophobic-Hydrophilic Surface and Janus Copper Foam Integrative System with Hierarchical Micro/Nanostructures. J. Colloid Interface Sci. 2020, 561, 730-740. 